\title{
159 Treatment of chronic frontal sinusitis
}

A Catheterization of the frontonasal duct should precede more radical operations.

B The Howarth operation combines treatment of frontal and maxillary sinuses.

C A drainage tube is left in the nose for several weeks after the Howarth operation.

D Obliteration of the sinus is an alternative to a drainage procedure.

E An osteoplastic flap is hinged superiorly.

\section{Aetiology of sinusitis in children}

A Antibody deficiency is a common cause.

B Dietary deficiency and poor social conditions are contributory.

C Bacteriology usually shows a pure growth of Haemophilus influenzae in chronic cases.

D Childhood exanthemata may initiate chronic sinusitis.

E Kartagener's syndrome consists of sinusitis, bronchitis, and congenital cyanotic heart disease.

\section{Sinusitis in children}

A The frontal sinus is poorly developed before the 5 th year.

$B$ In acute sinusitis, oedema of the cheeks and eyelids is commoner in children than adults.

C Pain is the principal clinical feature of chronic sinusitis in children.

D Chronic ethmoidal sinusitis should be treated by external ethmoidectomy.

E Adenoidectomy may improve the outlook in chronic maxillary sinusitis. 